\title{
Community Based Management of Severe Acute Malnutrition: The MSF Experience from an Urban Slum Setting in Bangladesh
}

\author{
Engy Ali ${ }^{*}$, Pascale Delchevalerie ${ }^{2}$, Zubair Shams ${ }^{3}$, Petra Alders ${ }^{2}$, Rony Zachariah ${ }^{1}$ \\ ${ }^{1}$ Luxembourg Operational Research Unit (LuxOR), Medical Department, Médecins Sans Frontières—Operational Centre Brussesl, \\ Luxembourg, Luxembourg \\ ${ }^{2}$ Medical Department, Médecins Sans Frontières-Operational Centre Brussels, Brussels, Belgium \\ ${ }^{3}$ Bangladesh Mission, Médecins Sans Frontières-Operational Centre Brussels, Dhaka, Bangladesh \\ Email: *engy.y.ali@gmail.com
}

How to cite this paper: Ali, E., Delchevalerie, P., Shams, Z., Alders, P. and Zachariah, R. (2020) Community Based Management of Severe Acute Malnutrition: The MSF Experience from an Urban Slum Setting in Bangladesh. Food and Nutrition Sciences, 11, 578-589.

https://doi.org/10.4236/fns.2020.116041

Received: May 25, 2020

Accepted: June 25, 2020

Published: June 28, 2020

Copyright (c) 2020 by author(s) and Scientific Research Publishing Inc. This work is licensed under the Creative Commons Attribution International License (CC BY 4.0).

http://creativecommons.org/licenses/by/4.0/

\begin{abstract}
Background: Until recently, the experience on implementing community based management of acute malnutrition (CMAM) among children has been largely based in African settings. While the government in Bangladesh is yet to scale up CMAM approach, there is still paucity of knowledge on the experience of CMAM within the complex milieu of an urban slum context. In Kamrangirchar slum, Dhaka, Bangladesh, this paper describes a CMAM programme performance and outcomes run by Médecins Sans Frontières (MSF)/Doctors without Borders, in light to performance indicators set by MSF and the Sphere minimum standards. Methods: This was a descriptive retrospective study using routinely collected programme data of children admitted with severe acute malnutrition between May 2010 and November 2011. Kamrangirchar is an urban slum of a large migrant population in Dhaka, Bangladesh. Results: There was a total of 640 new admissions, of whom 333 (52\%) were males. The median age was 18 months (Inter-quartile range (IQR) 12 - 41). 599 children had a reported nutritional outcome at discharge from ambulatory therapeutic feeding centre (ATFC), this included: cure rate of $69 \%$ with an average length of stay of $68.8(\mathrm{SD} \pm 46.0$ ) days and average weight gain of $3.8 \mathrm{~g} / \mathrm{kg} /$ day ( $\mathrm{SD} \pm 2.7$ ). The lost-to-follow-up rate was $18 \%$ and $5 \%$ reported to the programme that they will leave the slum and go back to their villages. These performance indicators did not meet the threshold level indicators set by MSF and Sphere standards. Conclusions: Our experience highlights the need for developing more adapted and contextualised indicators for assessing the performance of CMAM programmes in settings such as urban slums. Community engagement in the process of developing relevant standards is crucial. Nutrition humanitarian actors have a vital role
\end{abstract}


to collaborate with local authorities to contextualize and refine these standards.

\section{Keywords}

Severe Acute Malnutrition, Community Based Management, Nutrition Outcomes, Urban Slum, Bangladesh

\section{Introduction}

Children in slums are at high risk of severe acute malnutrition (SAM) and its associated morbidity and negative effects on their growth and cognitive development later in life [1]. In Bangladesh, an alarming rapid urbanization has forced poor rural population to migrate to urban areas, particularly in the capital city Dhaka, settling down in poor overcrowded informal slum settings. It is predicted that half of the population in Bangladesh will be living in urban settings by 2050 [2]. Children in overcrowded urban slums are particularly vulnerable to malnutrition and disease due to poor living condition and lack of access to essential basic services [3]. This is a major public health concern in Bangladesh which requires well adapted and targeted interventions, as the country has one of the highest burdens of children with acute malnutrition in the world [4]. According to the latest Bangladesh Demographic and Health Survey (BDHS) in $2014,14 \%$ of children under 5 were identified to be wasted [5].

The innovation of community-based management of acute malnutrition (CMAM) has been endorsed by the World Health Organization (WHO) to overcome the challenges of inpatient based approach such as low coverage, high mortality, and lost-to-follow-up [6] [7]. In brief, CMAM involves early detection of SAM children in the community: nutritional rehabilitation with ready-to-use therapeutic food (RUTF) (or other nutrient-dense foods) and ambulatory follow-up of children within the community. Children are only referred for inpatient care if they have medical complications. The widespread adoption of CMAM strategy has proved to improve geographic access, considerably reduce the burden on health services, foster community empowerment and enhances task-shifting initiatives [8] [9].

Assessing the performance of CMAM services supports on-going and future programmes through generating evidence on CMAM experiences. This is often performed by comparing to nutrition sector global performance indicators, including the widely used Sphere minimum standards [10]. The latter is a cornerstone reference in humanitarian practice, which provides universal benchmark to guide relief efforts in five sectors that dominate humanitarian settings, including nutrition, food aid, shelters, water and sanitation and health services [11]. Although the Sphere standards are claimed to be universal, they were largely based on experience in relief programmes, particularly in Africa. Therefore, the utility and relevance of the use of these standards in the complex milieu of urban slums 
remains questionable [12].

In Bangladesh, CMAM has been implemented at a limited scale by non-government organisations. In 2017, the government has prioritized the integration of CMAM into the health system, and developed national guideline for CMAM but has not been yet scaled up into the health system [13]. Médecins Sans Frontières (MSF)/Doctors without Borders was involved with implementing CMAM in Kamrangirchar, one of the largest urban slums in Dhaka, and the country, hosting a majority of migrant inhabitants from rural areas. To our knowledge, this was one of the first CMAM programme implemented in an urban slum in Bangladesh.

Although, various studies have tackled child malnutrition in Bangladesh, there is still paucity of knowledge on the experience of implementing CMAM in the complex setting of urban slum. As the government is yet to scale up CMAM in Bangladesh, it is pertinent to shed the light on some of the lessons learnt and challenges of implementing CMAM approach in a slum in Dhaka. Therefore, in Kamrangirchar slum, Bangladesh, this paper describes a CMAM programme performance and outcomes of children admitted with SAM, in light to performance indicators set by MSF and the Sphere standards.

\section{Methods}

\section{Study design}

This was a descriptive retrospective study using routine programme data of children with severe acute malnutrition (SAM) enrolled in MSF nutrition programme between May 2011 and November 2012.

\section{Study setting and population}

The CMAM programme was located in Kamrangirchar-an urban slum setting in Dhaka. It is the largest slum cluster in the Dhaka Metropolitan Area with an estimated population of 400,000 inhabitants living in an area of 3.1 $\mathrm{km}^{2}$. It is officially not part of Dhaka city and there were no governmental health structures in the slum. Provision of health care services was outsourced to non-governmental organisations. The majority of households in this highly dense slum are tin-sheds with shared latrine and cooking area. During the severe rainfall, water running through the latrines also makes the slum inhabitants more prone to water-borne diseases. The majority of inhabitants are migrant population from rural areas, and engaged in low income revenue activities, mostly waste recycling, ready-made garment (RMG) factories, informal manufacturing industry (e.g. tanneries) and in service jobs such as rickshaw pullers [14]. Most of the RMG factories are located in the vicinity of Dhaka which is a huge driving force for migration from rural to deprived informal urban slums in Dhaka including Kamrangirchar. The majority of the RMG workers are women with long working hours and low wage, so young children are often left alone or with their elder siblings for long hours [15].

MSF has been providing health services in Kamrangirchar through two primary health care (PHC) centres and all services were offered free of charge. The 
project targeted malnutrition among children aged less than five years. The management protocols of acute malnutrition are in line with the recommended WHO guidelines [16].

\section{Nutritional management of malnourished children}

Screening of children aged less than five years was done routinely at the PHC level for all children attending the MSF clinics. This involved mid-upper arm circumference measurements (MUAC) and weight for height $\mathrm{Z}$ score (WHZ). Screening was also done in the community (door-to-door) by a team of trained community health workers (CHWs) using the MUAC tape and verifying the presence of bilateral pitting oedema. Children were admitted to the nutrition programme if they were found with SAM (WHZ score $<-3$, MUAC $<115 \mathrm{~mm}$, bilateral nutritional oedema) or moderate acute malnutrition (MAM) (WHZ $\geq-3$ to $<-2$ ) with medical complications.

Initially, all the children were assessed clinically by a trained nurse for medical complications and underwent an appetite test for peanut-based RUTF (Plumpy' $\left.^{\prime} u^{(\mathbb{R}}\right)$. Children were admitted to the outpatient ambulatory therapeutic feeding centres (ATFC) if they passed the appetite test (the child ate a certain amount of RUTF in relation to child's body weight). Children who failed the appetite test or had severe medical complications were referred to a private hospital inpatient therapeutic feeding centre (ITFC) or admitted to the MSF day-care, according to severity of condition. On admission, all children received systematic empirical medical treatment (antibiotics, de-worming, measles vaccination, vita$\min$ A, iron and folic acid). The RUTF in the form of Plumpy'Nut and/or BP100 (high energy biscuit) were provided according to a standard protocol [17].

The slum was geographically divided into eight blocks, and the community based ATFCs 'satellite clinic' were offered in each block area on a weekly basis. The follow-up of the children and distribution of Plumpy'Nut was done on weekly basis. A collaborative partnership was established with the community and community leaders. The Community leaders offered the venues for ATFC and these included sites such as school rooms, office rooms, etc. The venues selected met certain criteria of having enough space and capacity/utilities to perform the follow up activities and provide nutrition education and cooking demonstrations.

During the follow up visits at ATFC, MUAC and weight were assessed by CHWs. Children were also assessed by a nurse and referred to the PHC for further clinical assessment, if they were found with any symptoms of illness (e.g. cough, fever, diarrhea).

The CHWs were directly responsible for the follow-up of children in the ATFC, distribution of Plumpy'Nut and continuing health promotion at the ATFC and in the community. The CHW team also traced loss-to-follow up children in the community, and inquired about the reasons, whenever possible. All CHWs were recruited from Kamrangirchar and received training sessions by an experienced Nutrition Nurse on the assessment using MUAC, weight, height 
(WHZ score). The team received regular refresher trainings on monthly basis to ensure the quality of anthropometric assessment. The team of CHWs was divided into five teams with three supervisors; each team was allocated to specific block(s). Transportation in the slum was done by means of an adapted Rickshaw. The nutrition programme was closed in mid-2012.

\section{Admission categories and nutritional outcomes}

Admission categories and nutritional outcomes were standardized as shown in the Box. Admission categories included a) new admission, b) readmissions and c) relapses. Nutritional outcomes included a) cure, b) loss-to-follow up, c) left Kamrangirchar, d) non-responder, e) transfer to hospital (deterioration rate) and $f$ ) death. The main outcomes were compared to the existing MSF [17] and Sphere minimum standards [10].

Box: Programme's admission categories and nutritional outcomes

Admission categories:

- New admission: A malnourished child admitted to the nutritional program for the first time

- Readmission: A child who is readmitted to the nutritional programme after being lost to follow-up for less than two months or having been transferred out to hospital.

- Relapse: A child who presents back to the nutritional programme with severe acute malnutrition within two months of discharge as cured.

\section{Nutritional outcomes}

- Cured: Attaining WHZ > -2 for two consecutive measurements, MUAC $>115 \mathrm{~mm}$, absence of oedema or acute medical complications.

- Lost-to-follow up: Absent for more than two consecutive weeks from the community and the ATFC follow up visits.

- Left Kamrangichar: Caretaker informing the programme that the child will leave the slum and return back to the village.

- Non responder: Child not attaining the target weight after more than 90 days of treatment after exclusion of underlying medical and social conditions.

- Transferred to hospital: Referral needed to inpatient health care due to deterioration.

- Died: Died whilst registered in the programme.

WHZ: weight for height $\mathrm{Z}$ score; MUAC: mid-upper arm circumference; ATFC: ambulatory therapeutic feeding centre.

\section{Statistical analysis}

Data during admission, follow-up and discharge of children admitted in the programme with SAM were entered into a Microsoft Excel spread sheet (Microsoft, Redwood, WA, USA). Data analysis was done using EpiData Manager (version 2.0.0.25, EpiData Association, Odense, Denmark).

\section{Ethics review}

Ethics approval was received from the MSF Ethics Review Board, Geneva, Switzerland and The Union Ethics Advisory Group, Paris, France.

\section{Results}

\section{Characteristics of the study population}

There was a total of 640 new admissions in the nutrition programme, of 
whom 333 (52\%) were males and 307 (48\%) were females (Table 1). The median age was 18 months (Inter-quartile range (IQR) 12 - 41). The majority 476 (74\%) of new admissions were through the routine screening at the PHC clinics, while 144 (23\%) were through community door-to-door screening and 20 (3\%) presented at the ATFC (self-referral by caretaker) (Table 1).

\section{Nutrition programme outcomes}

The nutrition outcomes of SAM children admitted to the programme in comparison to the ATFC performance indicators set by MSF and Sphere standards are shown in Table 2. Of all new admissions, 41 (6\%) children deteriorated and developed medical complications, and thus were transferred out to a private hospital ITFC. Excluding those transferred to ITFC, there was a total of 599 new admissions who had reported nutrition outcomes in the ATFC. The cure rate of these children was $69 \%$ and death rate was $1 \%$. The loss-to-follow up rate was $18 \%$ and $5 \%$ reported to the programme that they will leave the slum and go back to their villages. The average length of stay (ALS) was 68.8 days (SD \pm 46.0 ) and average weight gain (AWG) was $3.8 \mathrm{~g} / \mathrm{kg} /$ day $(\mathrm{SD} \pm 2.7)$. Notably, 44 (7\%) children did not recover after three months of treatment and were discharged as non-responders.

During the study period, 42 children were readmitted to the programme, of

Table 1. Baseline characteristics of new admissions to the ambulatory therapeutic feeding centres (ATFC) in Kamrangirchar, Dhaka, Bangladesh (May 2010-November 2011).

\begin{tabular}{cc}
\hline Characteristics & (n-\%) \\
Total new admissions & 640 \\
Male & $333(52)$ \\
Female & $307(48)$ \\
Age (months) & \\
$6-11$ & $134(21)$ \\
$12-23$ & $223(35)$ \\
$24-35$ & $83(13)$ \\
$36-59$ & $200(31)$ \\
Age in months (median-IQR) & $18(12-41)$ \\
Mode of admission & \\
PHC routine screening & $476(74)$ \\
Door-to-door screening & $144(23)$ \\
ATFC (self-referral) & $20(3)$ \\
Admission Criteria & \\
MUAC < 115 mm & $278(43)$ \\
WHZ $<-3$ & $352(55)$ \\
Oedema & $10(2)$ \\
\hline
\end{tabular}

IQR: inter-quartile range, PHC: primary health care; ATFC: ambulatory therapeutic feeding centre; MUAC: mid-upper arm circumference; WHZ: Weight for height $Z$ score. 
whom 35 (83\%) were readmitted after being transferred out to a hospital ITFC care and 7 (17\%) after being lost-to-follow-up (data are not shown in Table 2). Of the 412 children who were discharged as cured, $16(4 \%)$ presented with relapse during the study period of one year. The nutrition outcome of readmitted children and those presented with relapse are shown in Table 2.

\section{Discussion}

To our knowledge, this is one of the first descriptive studies showing the nutritional programme performance using a CMAM approach in an urban slum in Dhaka, Bangladesh. The programme cured and lost-to-follow-up rates, average weight gain and average length of stay did not meet the minimum performance indicators set by MSF and Sphere standards. The latter is based on experience in famine and relief programmes in Africa and largely used as proxy to monitor and evaluate relief programmes. This raises the pertinent question on whether such existing standards-are realistic and relevant to the complex urban context of malnutrition among migrant populations living in urban slums in Southern

Table 2. Nutritional outcomes of children admitted with severe acute malnutrition to the ambulatory therapeutic feeding centre (ATFC) in Kamrangirchar, Dhaka, Bangladesh (May 2010-November 2011).

\begin{tabular}{|c|c|c|c|}
\hline Nutritional Outcomes & $(\mathrm{n}-\%)$ & $\begin{array}{l}\text { MSF performance } \\
\text { indicators (ATFC) }\end{array}$ & $\begin{array}{c}\text { Sphere } \\
\text { performance } \\
\text { indicators }^{\mathrm{b}}\end{array}$ \\
\hline New admissions & $599^{\mathrm{a}}$ & & \\
\hline Cured & $412(69)$ & $>80 \%$ & $>75 \%$ \\
\hline Lost-to-follow up & $105(18)$ & $<15 \%$ & $<15 \%$ \\
\hline Left Kamrangirchar & $32(5)$ & - & - \\
\hline Died & $6(1)$ & $<3 \%$ & $<10 \%$ \\
\hline Non-responder & $44(7)$ & - & - \\
\hline \multicolumn{4}{|l|}{ Treatment-response indicators (cured) } \\
\hline Average weight gain (g/kg/day) (SD) & $3.8( \pm 2.7)$ & $5-10$ & $>8$ \\
\hline Average length of stay (days) (SD) & $68.8( \pm 46.0)$ & $<45$ & $30-40$ \\
\hline Readmissions & 42 & & \\
\hline Cured & $28(67)$ & & \\
\hline Lost-to-follow up & $8(19)$ & No indicators & No indicators \\
\hline Transferred to hospital & $6(14)$ & & \\
\hline Relapses & 16 & & \\
\hline Cured & $15(94)$ & & \\
\hline Lost-to-follow up & - & No indicators & No indicators \\
\hline Transferred to hospital & $1(6)$ & & \\
\hline
\end{tabular}

${ }^{a}$ total excluding those transferred out to private hospital ITFC; ${ }^{\text {b }}$ for therapeutic care; ATFC: ambulatory therapeutic feeding centre. 
Asia. A number of findings merit discussion.

First, the programme had an overall recovery rate of $69 \%$ which is below the $75 \%$ minimum set by The Sphere standards and the $80 \%$ by MSF. This programme cure rate was in line with a large scale CMAM project in Mumbai slums, India that reported a 56\% cure rate [18], and lower than the $91.9 \%$ cure rate reported in another CMAM programme in rural Southern Bangladesh [1]. The underlying reasons for the low cure rate in our study lies in the fact that nearly one-in-four new admissions were either "lost to follow-up" or had actually "left Kamrangirchar" to another setting, and thus negatively influenced the achievable cure rate. Loss-to-follow up was high despite the presence of a well-supported and resourced community follow-up and tracing system. Moreover, CHWs were operating within a relatively small geographic area of four $\mathrm{km}^{2}$. One might therefore think that such losses could not be avoided considering the predominantly migrant setting. It might thus be realistic to assume that $15 \%-20 \%$ of all new admissions would end up as a combination of "lost to follow-up" and "left Kamrangirchar" as observed in our experience. This then begs the question about what the target cure rate of an ATFC should be in such a slum setting? If the acceptable threshold for death is maintained at $<10 \%$, then an achievable operational threshold for the cure rate in such a setting would be $70 \%$ in a best case scenario.

An additional limitation of existing thresholds is the lack of any indicator for deterioration rate expressed as transfer out to hospital for inpatient care. Such rates are subject to factors such as the baseline severity of malnutrition, the medical condition of the child and the capacity and quality of clinical management. High transfer rates to inpatient facilities will negatively influence the cure rate and programme performance, these need to be monitored.

Second, beside a considerable proportion of the population in Kamrangirchar are migrants in transitory phase, the high lost to follow-up rate observed in the programme, might be due to household factors. The majority of mothers were form the core of "daily waged workers", mainly in RMG factories. Such work requires working long hours with limited availability of extended family [15], making attendance at the scheduled weekly follow-up appointments difficult. Adaptation of ATFC open hours and follow-up schedule (e.g. fortnightly visits) might be needed in this kind of setting. We also observed temporary displacements of population for social and culture events. This included migration during the harvest season or travel of pregnant women back to home-villages for delivery. The latter is a distinct group which needs to be distinguished from the classical "lost to follow-up" category as it involves formal contact with the programme staff with a clear justification for discontinuation of the nutritional care for their children. Such an outcome is so far unreported in the literature but merits being included in standard nutritional outcomes for such settings.

Third, the ALS exceeded by approximately 50\% the existing MSF threshold of 45 days and this could be attributable to insufficient average weight gain in the 
cohort. This could be strongly linked to the mediocre or poor compliance to the provided peanut based RUTF which proved to have low acceptability among caretakers and CHWs in our programme [19]. Care takers availability and food sharing within the family could be also contributing factors to this long ALS. These factors need to be explored in order to set an appropriate threshold for the ALS in such setting.

Fourth, we used fixed community outreach clinics to offer ATFC services despite the small coverage area of three $\mathrm{km}^{2}$. This was justified on the basis that the small geographic slum area is characterized by winding and congested roads and a dense population making travel difficult for mothers and children. In retrospect, the down-side of fixed clinics is that it requires displacement from home, time loss and associated indirect costs albeit to a lesser extent than hospital based care. A study from rural Bangladesh demonstrated that well-trained and supervised CHWs were able to effectively manage uncomplicated cases of SAM. This CMAM approach was centred mainly on the CHW identifying SAM children in the community and providing follow-up and treatment at home. The approach was also well accepted by the care takers [20]. A similar approach would seem the right way to go forward in the slums.

Fifth, in a setting where stunting has been prevalent for decades, there is a general perception that malnutrition is not a "disease". As such, attention is given to these children only when an associated morbidity develops. This may also explain why seven of ten children admitted to the nutritional programme came in through the routine screening at the PHC. A qualitative study from a rural context in Bihar, India, showed that undernutrition was seen not as a disease per se but as a result of different disease conditions and was often related to witchcraft [21]. It is crucial to understand these aspects which might have substantial impact on the uptake and attrition in the CMAM programme. Increased community awareness and empowerment is needed to address this issue. New messages and approaches to communicate effectively in such context need to be also discovered.

Sixth, the relapse rate was $4 \%$, despite an important focus in the programme on nutrition education and cooking demonstration. Through anecdotal data of health promotion team during routine discussions with mothers and caretakers, we discovered that low purchasing power of families is an obstacle to adherence to nutrition counselling: even if mothers knew what is good for their child, they often could not afford it. On the other hand, the definition of relapse is still poorly understood and undefined [22]. A performance indicator for deciding acceptable levels of relapse also needs attention.

Finally, Community engagement is a corner-stone for CMAM implementation; it includes all community components of CMAM as well as interactions with the community. It seeks beyond community sensitisation and outreach activities (e.g. early case-finding, referral of identified children, home follow-up visits), towards establishing community ownership and collaboration in all as- 
pects of CMAM services [23]. Although efforts were made to ensure community engagement in Kamrangirchar, some of the indicators of these activities were not fully or systematically monitored in the programme. Similarly, there are no standardised indicators of community engagement activities globally and therefore guidance on monitoring community engagement and examples of good practices in slum settings are still lacking in the literature.

The study has a number of limitations. The CMAM project in Kamrangirchar was closed in 2012 for operational reasons, but detailed data was available only till November 2011. Although, the study used historical programme data from 2010-2011, this data is relevant to guide future CMAM programme in similar settings in Bangladesh. To our knowledge, there is still no published literature on a CMAM performance from such a unique urban slum in Dhaka, Bangladesh. We assume the living conditions and the demography of the slum have not markedly changed, and thus the study results would be helpful to guide the government effort to scale up CMAM and integrate it to primary health structures.

\section{Conclusion}

In conclusion, our experience from an urban slum setting in Bangladesh has highlighted the need to develop more adapted and contextualized indicators for assessing the performance of CMAM programme in settings such as urban slums. Community engagement in the process of developing relevant standards to their context is crucial. Nutrition humanitarian actors have a vital role to collaborate with local authorities to contextualize and refine these standards.

\section{Funding}

The study was funded by MSF-Brussels Operational Centre.

\section{Acknowledgments}

We thank the MSF field team working in Kamrangirchar and in particular the $\mathrm{CHW}$ team. We are also grateful to the community leaders in Kamrangirchar for the collaboration they offered to MSF.

\section{Authors Contribution}

EA, RZ, PD, PA were involved in the conception and the design of the study. EA and RZ wrote the study protocol and all co-authors contributed to its improvement. Data analysis was done by EA. The first draft of the manuscript was written by EA and RZ and was critically reviewed by all co-authors. All co-authors reviewed and approved the final version.

\section{Conflicts of Interest}

The authors declare no conflicts of interest regarding the publication of this paper. 


\section{References}

[1] Puett, C., Sadler, K., Alderman, H., Coates, J., Fiedler, J.L. and Myatt, M. (2013) Cost-Effectiveness of the Community-Based Management of Severe Acute Malnutrition by Community Health Workers in Southern Bangladesh. Health Policy Plan, 28, 386-399. https://doi.org/10.1093/heapol/czs070

[2] Ahsan, K.Z., Arifeen, S.E., Al-Mamun, Md.A., Khan, S.H. and Chakraborty, N. (2017) Effects of Individual, Household and Community Characteristics on Child Nutritional Status in the Slums of Urban Bangladesh. Archives of Public Health, 75, 9. https://doi.org/10.1186/s13690-017-0176-X

[3] Lilford, R.J., Oyebode, O., Satterthwaite, D., Melendez-Torres, G.J., Chen, Y.-F., Mberu, B., et al. (2017) Improving the Health and Welfare of People Who Live in Slums. The Lancet, 389, 559-570. https://doi.org/10.1016/S0140-6736(16)31848-7

[4] Fakir, A.M.S. and Khan, M.W.R. (2015) Determinants of Malnutrition among Urban Slum Children in Bangladesh. Health Economics Review, 5, 22.

https://doi.org/10.1186/s13561-015-0059-1

[5] Bangladesh Demographic and Health Survey 2014. https://dhsprogram.com/pubs/pdf/FR311/FR311.pdf

[6] World Health Organisation (WHO) (2009) WHO Child Growth Standards and the Identification of Severe Acute Malnutrition in Infants and Children: A Joint Statement by the World Health Organization and the United Nations Children's Fund. https://apps.who.int/iris/bitstream/handle/10665/44129/9789241598163_eng.pdf?se quence $=1$

[7] World Health Organisation (WHO) (2018) Joint Child Malnutrition Estimates-Levels and Trends: UNICEF-WHO the World Bank Group. https://www.who.int/nutgrowthdb/2018-jme-brochure.pdf?ua=1

[8] Collins, S., Sadler, K., Dent, N., Khara, T., Guerrero, S., Myatt, M., et al. (2006) Key Issues in the Success of Community-Based Management of Severe Malnutrition. Food and Nutrition Bulletin, 27, S49-S82. https://doi.org/10.1177/15648265060273S304

[9] Ashworth, A. (2006) Efficacy and Effectiveness of Community-Based Treatment of Severe Malnutrition. Food and Nutrition Bulletin, 27, S24-S48. https://doi.org/10.1177/15648265060273S303

[10] Humanitarian Charter and Minimum Standards in Humanitarian Response (2018) The Sphere Handbook.

[11] Patel, R.B. and Chadhuri, J. (2019) Revisiting the Sphere Standards: Comparing the Revised Sphere Standards to Living Standards in Three Urban Informal Settlements in Nairobi, Kenya. Journal of International Humanitarian Action, 4, 6. https://doi.org/10.1186/s41018-019-0054-y

[12] Dufour, C., Geoffroy, V., Maury, H. and Grünewald, F. (2004) Rights, Standards and Quality in a Complex Humanitarian Space: Is Sphere the Right Tool? Disasters, 28, 124-141. https://doi.org/10.1111/j.0361-3666.2004.00248.x

[13] Ministry of Health and Family Welfare, Government of the People's Republic of Bangladesh, 4th Health Nutrition and Population Sector Program (HNPSP). http://www.mohfw.gov.bd/index.php?option=com_content\&view=article\&id=78\&I temid=59\&lang=en

[14] van der Heijden, J., Gray, N., Stringer, B., Rahman, A., Akhter, S., Kalon, S., et al. (2019) "Working to Stay Healthy", Health-Seeking Behaviour in Bangladesh's Urban Slums: A Qualitative Study. BMC Public Health, 19, Article ID: 600. 
https://doi.org/10.1186/s12889-019-6750-0

[15] ChildHope Projects (2018) Protecting the Children of Bangladeshi Garment Workers.

https://www.childhope.org.uk/assets/uploads/assets/364300\%20ChildHope\%20Proj ects\%20-\%20Bangladesh\%20-\%20Nagorik\%20Uddyog.pdf

[16] World Health Organisation (WHO) (2007) Community-Based Management of Severe Acute Malnutrition: A Joint Statement by the World Health Organization, the World Food Programme, the United Nations System Standing Committee on $\mathrm{Nu}$ trition and the United Nations Children's Fund.

https://www.who.int/nutrition/topics/Statement_community_based_man_sev_acut e_mal_eng.pdf

[17] Nutritional and Medical Protocol for Treatment of Severe Malnutrition-Outpatient Children from 6 Months to 10 Years: MSF OCB, May 2015.

http://ocbmed.msf.be/\#./english/medical-thematics/nutrition/index.html

[18] Goudet, S., Jayaraman, A., Chanani, S., Osrin, D., Devleesschauwer, B., Bogin, B., et al. (2018) Cost Effectiveness of a Community Based Prevention and Treatment of Acute Malnutrition Programme in Mumbai Slums, India. PLoS ONE, 13, e0205688. https://doi.org/10.1371/journal.pone.0205688

[19] Ali, E., Zachariah, R., Dahmane, A., Van den Boogaard, W., Shams, Z., Akter, T., et al. (2013) Peanut-Based Ready-to-Use Therapeutic Food: Acceptability among Malnourished Children and Community Workers in Bangladesh. Public Health Action, 3, 128-135. https://doi.org/10.5588/pha.12.0077

[20] Puett, C., Coates, J., Alderman, H. and Sadler, K. (2013) Quality of Care for Severe Acute Malnutrition Delivered by Community Health Workers in Southern Bangladesh. Maternal \& Child Nutrition, 9, 130-142. https://doi.org/10.1111/j.1740-8709.2012.00409.x

[21] Burtscher, D. and Burza, S. (2015) Health-Seeking Behaviour and Community Perceptions of Childhood Undernutrition and a Community Management of Acute Malnutrition (CMAM) Programme in Rural Bihar, India: A Qualitative Study. Public Health Nutrition, 18, 3234-3243. https://doi.org/10.1017/S1368980015000440

[22] Stobaugh, H.C., Mayberry, A., McGrath, M., Bahwere, P., Zagre, N.M., Manary, M.J., et al. (2019) Relapse after Severe Acute Malnutrition: A Systematic Literature Review and Secondary Data Analysis. Maternal \& Child Nutrition, 15, e12702. https://doi.org/10.1111/mcn.12702

[23] Gray, N., Bedford, J., Deconinck, H. and Brown, R. (2014) Community Engagement: The "C" at the Heart of CMAM. CMAM Forum Technical Brief.

\section{Abbreviations}

ATFC Ambulatory therapeutic feeding centre

CMAM Community management of acute malnutrition

ITFC Inpatient therapeutic feeding centre

MUAC Mid-upper arm circumference

RUTF Ready to use therapeutic food

SAM Severe acute malnutrition

WHZ Weight-for-height z-score 